\title{
Fractured reservoir modeling by discrete fracture network and seismic modeling in the Tarim Basin, China
}

\author{
Sam Zandong Sun ${ }^{*}$, Zhou Xinyuan², Yang Haijun², Wang Yueying', Wang \\ Di $^{1}$ and Liu Zhishui ${ }^{1}$
}

${ }^{1}$ Laboratory for Integration of Geology \& Geophysics, China University of Petroleum, Beijing 102249, China

${ }^{2}$ Research Institute of Exploration \& Development, PetroChina Tarim Oilfield Company, Korla 841000, China

(C) China University of Petroleum (Beijing) and Springer-Verlag Berlin Heidelberg 2011

\begin{abstract}
Fractured reservoirs are an important target for oil and gas exploration in the Tarim Basin and the prediction of this type of reservoir is challenging. Due to the complicated fracture system in the Tarim Basin, the conventional AVO inversion method based on HTI theory to predict fracture development will result in some errors. Thus, an integrated research concept for fractured reservoir prediction is put forward in this paper. Seismic modeling plays a bridging role in this concept, and the establishment of an anisotropic fracture model by Discrete Fracture Network (DFN) is the key part. Because the fracture system in the Tarim Basin shows complex anisotropic characteristics, it is vital to build an effective anisotropic model. Based on geological, well logging and seismic data, an effective anisotropic model of complex fracture systems can be set up with the DFN method. The effective elastic coefficients, and the input data for seismic modeling can be calculated. Then seismic modeling based on this model is performed, and the seismic response characteristics are analyzed. The modeling results can be used in the following AVO inversion for fracture detection.
\end{abstract}

Key words: Fractured reservoir, Discrete Fracture Network (DFN), equivalent medium, seismic modeling, azimuth-angle gathers

\section{Introduction}

The Tarim Basin is a large-scale superimposed basin. For example, the Tazhong area has undergone multi-stage tectonic movements and crust uplifting. The strata have suffered strong weathering, denudation and corrosion (Wei et al, 2000; Luo et al, 2005). Therefore, many unconformities are formed. Moreover, the bedrock of the Tazhong carbonate reservoirs is characterized by low porosity and low permeability, and secondary storage space such as fractures, pores and caves are well developed (Yang et al, 2000). As a result, the reservoirs have strong heterogeneity. Carbonate reservoir prediction is a difficult problem, throughout the whole world. However, the Tarim Basin is the energy base in China and exploration is of great importance to the national economy and people's livelihood. So reservoir prediction in the carbonate rock of the Tarim Basin is more significant than ever.

Seismic exploration makes use of the relationship between the propagation characteristics of the seismic wave and the physical properties of the strata. Reservoirs usually show anisotropic characteristics when fractures exist. Therefore,

\footnotetext{
* Corresponding author. email: samzdsun@yahoo.com
}

Received March 18, 2011 we should investigate the seismic wave field based on the assumption of anisotropy. When we carry out the seismic wave forward modeling, first, the fracture models should be built according to geological statistics based on the geologic data, seismic data and well logging data. Secondly, through the analysis of the fracture models, the fractured reservoir can be equaled to a specific anisotropic model and the elastic coefficients can be computed. Finally, we can model the seismic wave field. The modeling result can not only help us to study the seismic wave characteristics and provide the guidance to field acquisition, but also can be utilized for AVO inversion to extract fracture information. So the seismic wave modeling is an important part.

\section{A research concept for fractured reservoir prediction}

Fractured reservoirs are an important type of oil and gas reservoir in the Tarim Basin and they show strong anisotropy. When AVO inversion is carried out to predict fracture development, fractures are often viewed as the Horizontal Transverse Isotropy (HTI) medium. However, the real fracture system is rather complicated. So the theoretical inversion results are very likely to contain some errors. In 
this paper, an integrated reservoir prediction workflow is put forward and is shown in Fig. 1. The research concept is listed as follows. Based on the geological, well logging and seismic data, the DFN model is set up to predict the spatial distribution characteristics of the fractures and some parameters describing the development of fractures are output. According to the DFN model established, we can build the corresponding effective anisotropic model and compute the effective elastic parameters. Seismic modeling can be then performed to study the seismic response characteristics of the fractured reservoir. Finally, the modeling results are used to perform AVO inversion. The fracture information can be exacted from AVO inversion, and is compared to the fracture model to analyze the inversion errors.

During the process of fractured reservoir prediction, seismic modeling plays a bridging role. However, before the seismic modeling is carried out, an effective anisotropic model is needed, which is often difficult to build in view of the complicated fracture system in the Tarim Basin. Therefore, the DFN method is applied in this paper for fractured reservoir modeling, and it will be discussed in detail in this paper.

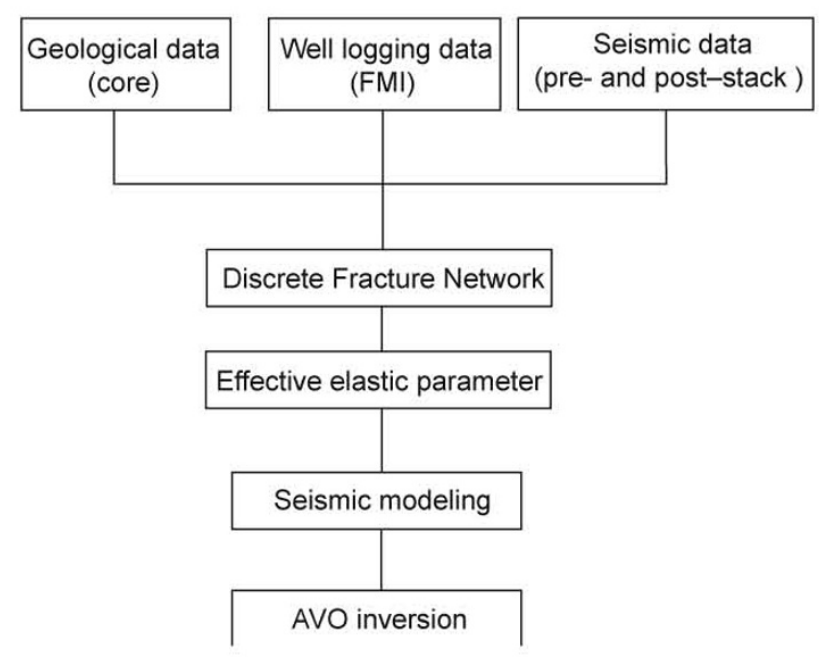

Fig. 1 Integrated workflow of fractured reservoir prediction

\section{Fractured reservoir modeling by DFN}

\subsection{Data analysis}

Geological, well logging and seismic data are essential for fractured reservoir modeling, and they can reflect fracture development at different scales. The geological ways to describe the fractures include structural evolution, tectonic stress analysis and cores. The well logging data is mainly Formation MicroScanner Image (FMI) and the seismic data include the seismic sections, faults and horizons. However, the most direct way is to use the cores and FMI. The cores display the subsurface fractures, while the FMI gives information about the fractures around the well. Besides providing direct and continuous images around the well, FMI can also be used to orient the horizons and fractures. We select the TZ45 area as an example.

\subsubsection{Analysis of cores}

There are three cored wells TZ45, TZ451 and TZ86 in the TZ45 area. According to the mechanical properties, both shear fractures and tensional fractures develop in the Lianglitage formation and there are rare compression-torsion fractures. Shear fractures are always filled with asphalt, mud, calcite or pyrite (Wang et al, 2002; Zhu et al, 2005). Tensional fractures are mainly filled with calcite. From the cores and slices, the reservoir storage spaces can be classified into three types: pore, cave and fracture (Zhao et al, 2007), and we can identify two kinds of caves (Wang et al, 2004): one is formed in the submarine diagenesis stages or the shallow buried stages (Fig. 2), the other is formed by deep hydrothermal karsts in which the early fractures are corroded unequally. The shear fractures and tensional fractures are corroded and filled with calcite and asphalt (Wang et al, 2007).

\subsubsection{Analysis of FMI}

There are four wells with FMI data in TZ45 area: TZ45, TZ86, TZ88 and ZG17. Fig. 3 shows the FMI data of TZ45 and TZ86 wells. After the analysis, we can find the rules as follows: the fracture-dip directions of the Liang1 formation in well TZ45 are mostly EW, and those of Liang2 are mainly NE-SW and EW. The fracture-dip directions of Liang2 in well TZ86 are complex and multi-directional, while those of Liang3 are mainly NS and NW-SE.

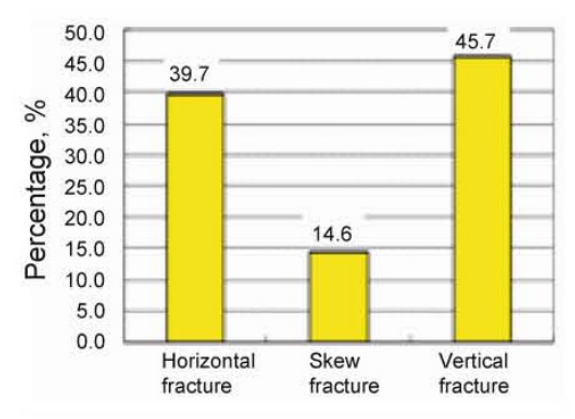

(a) Fracture occurrence

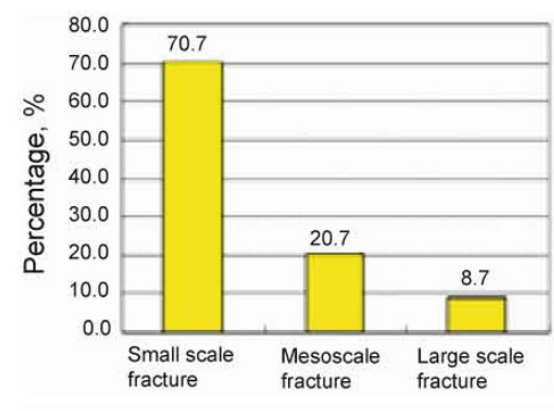

(b) Fracture size

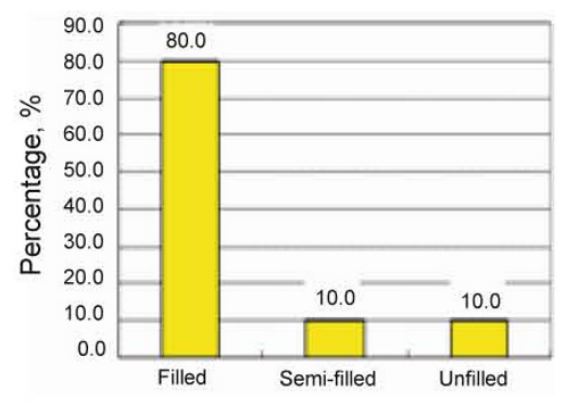

(c) Fracture fillings

Fig. 2 Statistical histogram of middle and upper Ordovician limestone-reservoir fracture characteristics 


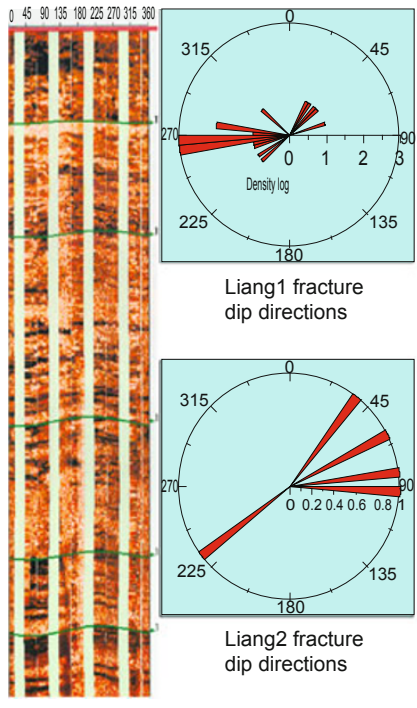

(a) Statistics of the TZ45 FMI

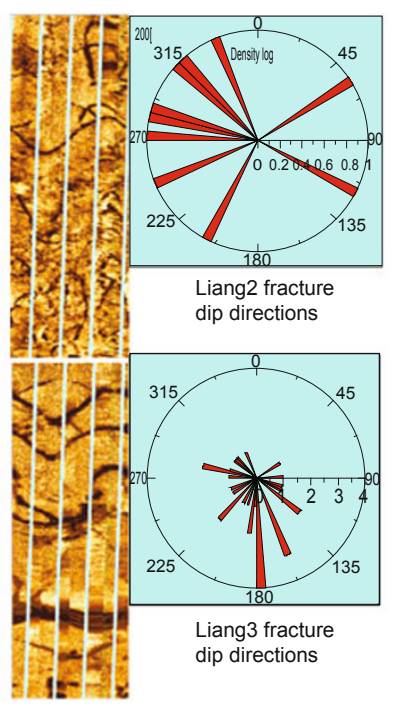

(b) Statistics of the TZ86 FMI

Fig. 3 Statistics of the FMI in the TZ45 area

\subsection{Fracture analysis}

After the geological model is set up, the cores, FMI and fault information from seismic data are needed. According to the geological statistics, the information such as fracture orientation and dip angle are analyzed. The fracture analysis method includes single-well analysis and multi-well integrated analysis.

The fracture analysis result of well TZ45 is shown in Fig. 4(a), and the dip direction is recognized to be mainly NE$\mathrm{SW}$. Both high angle and low angle fracture exist in well TZ45. The high angle ranges from 65 degrees to 85 degrees, while the low angle ranges from 5 degrees to 20 degrees. An integrated fracture analysis from wells TZ45, TZ86 and TZ88 is shown in Fig. 4(b). Two groups of fractures can be observed in the TZ45 area. One dip direction is NE-SW, with the dip angle ranging from 45 degrees to 85 degrees, and the other is NW-SE, with the dip angle ranging from 10 degrees to 75 degrees.

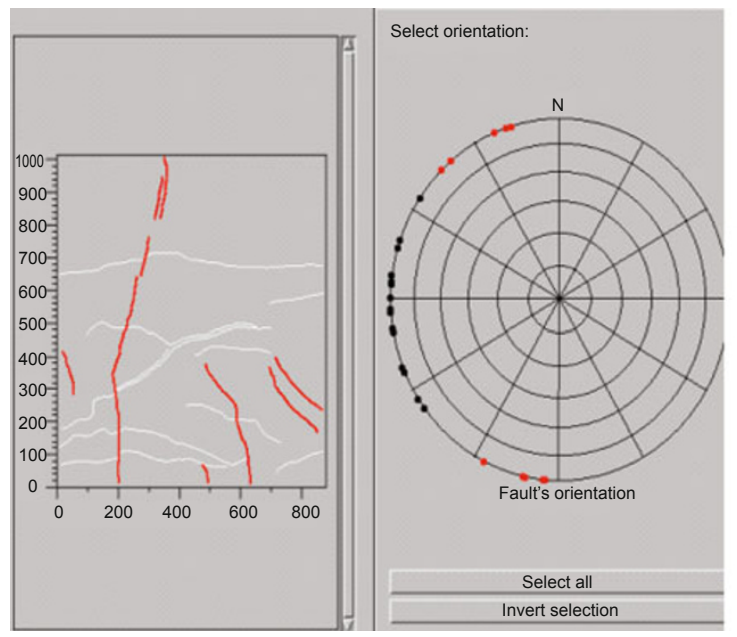

(a) D1 Fault

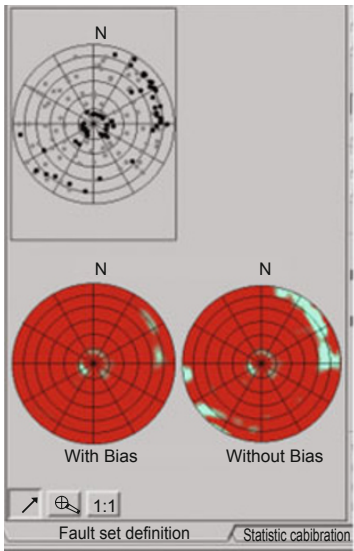

(a) Fracture analysis of well TZ45

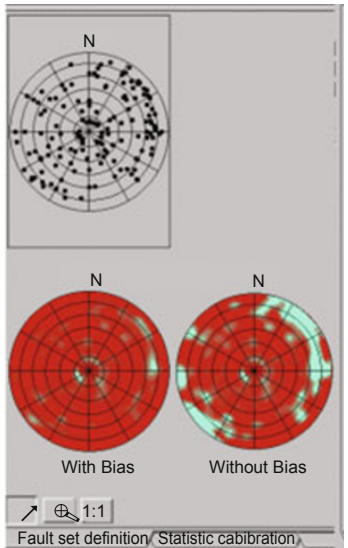

(b) Integrated fracture analysis (TZ45, TZ86, TZ88)
Fig. 4 Fracture analysis in the TZ45 area

\subsection{Fault analysis}

According to the seismic interpretation results, two groups of faults are developed in TZ45 area: NE-SW (D1) and NWSE (D2), as shown in Fig. 5. For fault D1, its strike is 204 degrees, and the grid dimension is 1.06 . The average length of the fault is $1020 \mathrm{~m}$, and the dip Fischer coefficient is 2.6. For fault D2, its strike is 85 degrees, and the grid dimension is 1.23 . The average length of the fault is $1193 \mathrm{~m}$, and the dip Fischer coefficient is 2.9. If D1 and D2 can be assumed to be vertical faults, D2 is cut by D1.

\subsection{Discrete fracture network (DFN) modeling}

Through analyzing the FMI data of the three wells and the fault information, the spatial distribution characteristics can be predicted and the DFN model can be built up. The DFN model of the block fractures around well TZ45 is shown in Fig. 6. The fractures with dip direction of NW-SE are well developed, which are consistent with the FMI interpretation results.

Based on the DFN model established, the fracture parameters such as fracture azimuth, strike, orientation and

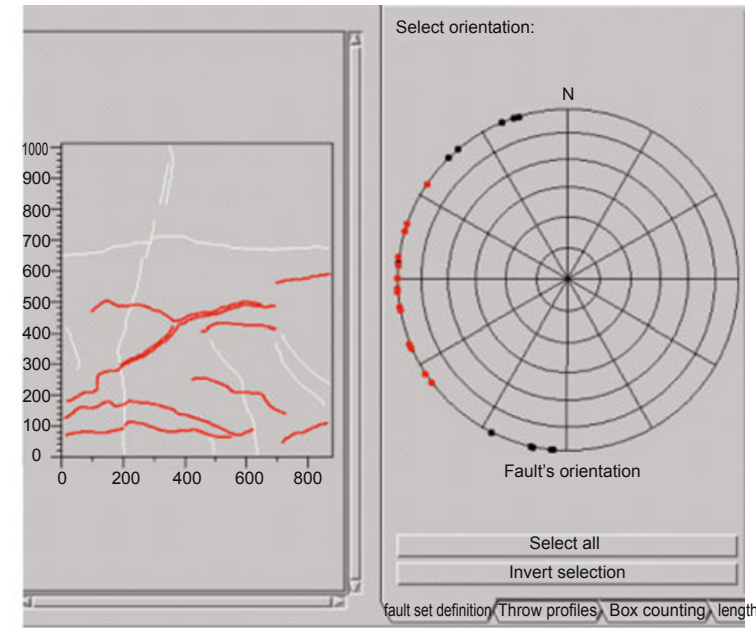

(b) D2 Fault

Fig. 5 Fault analysis in the TZ45 area 


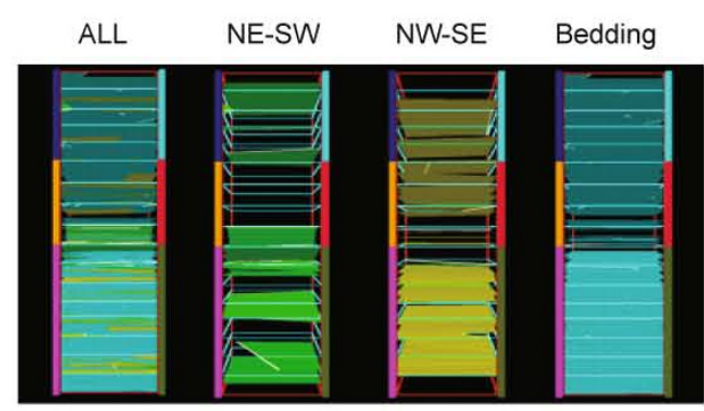

Fig. 6 DFN model around well TZ45

density can be acquired. These fracture parameters can be used to calculate effective elastic coefficients. The steps for extracting the fracture parameters are listed as follows.

(1) Determination of the coordinate system

The coordinate system defined in the DFN model follows the left-hand rule (Fig. 7) and all the fracture parameters are output in this system. The fracture can be described by two angles: azimuth angle $\theta$, calculated in clockwise direction, and dip angle $\varphi$. At the same time, it can be represented by Cartesian coordinates. The normal vector of the fracture has an intersection point with the fracture plane, its Cartesian coordinates $\left(N_{x}, N_{y}, N_{z}\right)$ can be used to represent the fracture angle and azimuth. These two description methods can be converted one to another.

(2) Determination of fracture shape

In the DFN model, the fracture is seen as a polygon and two principles are employed in the determination of the fracture shape. Firstly, the nodes of the polygon are assumed to be placed in clockwise or anticlockwise sequence. Secondly, the polygon is regarded as a convex polygon, rather than a concave polygon.

(3) Area calculation

The geometric center is determined firstly, and then the polygon is divided into different triangles. The area of the polygon can be obtained through the sum of the areas of the triangles, as shown in Fig. 8.

(4) Fracture radius calculation

Two methods can be used to calculate the fracture radius: the circumference method and the area method. If the polygon can be seen as a circle, once its circumference or the area is known, the radius can be easily worked out.

(5) Fracture grouping criteria

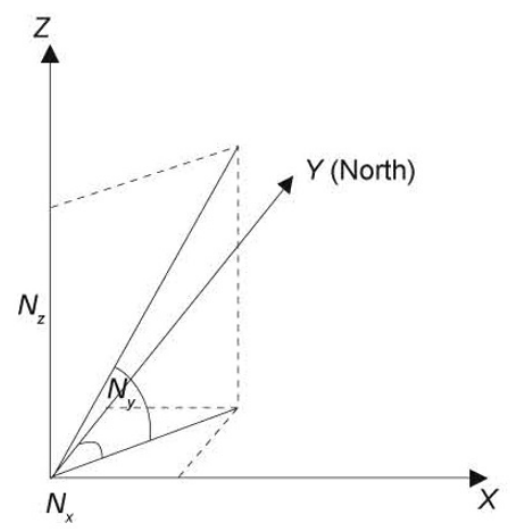

Fig. 7 Fracture azimuth description

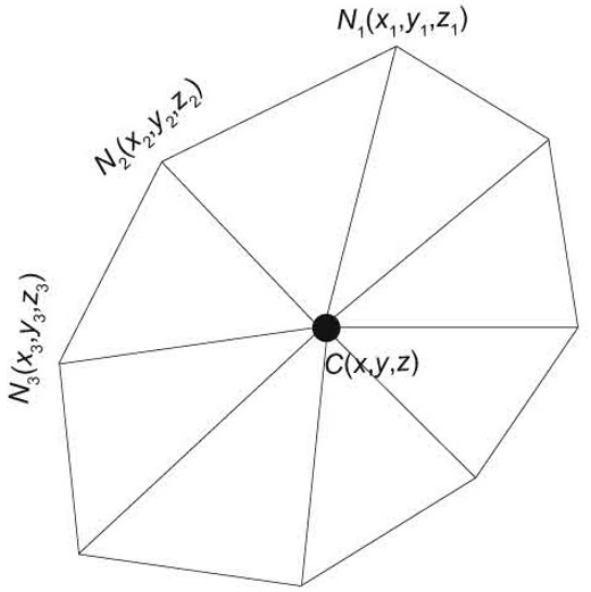

Fig. 8 Area calculation method

When the fractures are grouped, the fracture size is ignored. The fractures are projected to a Schmidt Coordinate System, and they can be divided into different groups according to the azimuth. For example, the azimuth angle interval is set to be 10 degrees and the dip angle interval is set to be 10 degrees. Thus, the fractures are divided into $36 \times 9$ groups. For simple fractures, whose strike direction and dip angle are within limited ranges, they can be seen as one group. However, for complex fractures, whose strike direction and dip angle are dispersedly distributed, they can be divided into several groups based on dominant fractures.

(6) Fracture density calculation

Fracture density is an important parameter of a fracture system. It refers to the number of fractures vertically passing through the fracture plane.

\subsection{Calculation of the effective elastic coefficients}

The purpose of building a DFN model is to establish the effective anisotropic model and calculate effective elastic coefficients. The Hudson effective model is adopted in this paper. The formula to compute the elastic coefficients is given under some assumptions. It is assumed that the medium contains directed and sparse fractures which are much smaller in size than the wavelength. These fractures are separated from each other, and the bulk modulus of the fluid is smaller than that of the background. According to Hudson's perturbation principle (Crampin, 1984; Hudson, 1986; Tsvankin, 2005), the effective elastic coefficients can be calculated from the Eq. (1):

$$
C_{i j k l}=C_{i j k l}^{0}+C_{i j k l}^{1}+C_{i j k l}^{2}
$$

where, $C^{0}$ is the elastic coefficient of the background media, and $C^{1}$ is the first-order disturbance quantity caused by groups of fractures, and $C^{2}$ is the second-order disturbance quantity caused by all fractures. $C^{1}$ and $C^{2}$ can be written respectively as Eqs. (2), (3) and (4):

$$
\begin{aligned}
& C^{1}=\sum_{i=1}^{m} C_{i}^{1} \\
& C_{i j p q}^{2}=C_{i j r s}^{1} \chi_{r s k l} C_{k l p q}^{1}
\end{aligned}
$$




$$
\chi_{r s k l}=\left[\delta_{r k} \delta_{s l}\left(4+\beta^{2} / \alpha^{2}\right)-\left(\delta_{r l} \delta_{s k}+\delta_{r s} \delta_{k l}\right)\left(1-\beta^{2} / \alpha^{2}\right)\right] /(15 \mu)
$$

where, $\alpha, \beta$ and $\mu$ respectively represents the $v_{\mathrm{p}}, v_{\mathrm{s}}$ and shear modulus of the background medium. $\delta_{i j}$ is the Kronecker symbol. During the calculation process of $C^{1}$ and $C^{2}$, fracture parameters such as fracture density derived through DFN will be used. In this way, elastic parameters can be worked out eventually.

\section{Seismic modeling of a fractured reservoir in the Tarim Basin}

\subsection{Calculation of elastic coefficients}

By using the real geological, well logging and seismic data in the Tarim Basin, the DFN model is built. The fracture parameters are then derived from the DFN and are listed in Table 1. These parameters will be used to calculate the equivalent elastic coefficients.

Table 1 Fracture parameters obtained from DFN model

\begin{tabular}{ccccccc}
\hline $\begin{array}{c}\text { Fracture } \\
\text { radius }\end{array}$ & $\begin{array}{c}\text { Aspect } \\
\text { ratio }\end{array}$ & Density & $N_{x}$ & $N_{y}$ & $N_{z}$ & $\begin{array}{c}\text { Fracture } \\
\text { group }\end{array}$ \\
\hline 65.19 & 0.001 & 0.083 & 0.017912 & -0.07679 & 0.996886 & 1 \\
63.83 & 0.001 & 0.35 & -0.08912 & -0.9779 & 0.189109 & 2 \\
60.22 & 0.001 & 0.067 & -0.67422 & -0.5059 & -0.53805 & 3 \\
62.91 & 0.001 & 0.09 & -0.17247 & -0.15133 & 0.973321 & 4 \\
61.16 & 0.001 & 0.28 & -0.92245 & -0.26419 & 0.281571 & 5 \\
63.97 & 0.001 & 0.08 & -0.23245 & -0.12499 & -0.96454 & 6 \\
\hline
\end{tabular}

Based on the fracture parameters obtained from the DFN model analysis, equivalent elastic coefficients are calculated according to the Hudson theory. The elastic coefficients are shown in Eq. (5). From the elastic coefficient matrix we can get the following conclusion: the fractures in this study area are complex and is not the standard HTI or TTI (Tilted Transverse Isotropy) model, and here we define it as a quasi TTI model. On the basis of this elastic coefficient matrix, we conduct the wave field forward modeling using the finite difference method. The model which contains two horizontal layers is designed. The first layer is the quasi TTI medium, and the second layer is isotropic, where the P-wave velocity is $3,000.0 \mathrm{~m} / \mathrm{s}, \mathrm{S}$-wave velocity is $1,500.0 \mathrm{~m} / \mathrm{s}$ and the density is $2,000.0 \mathrm{~kg} / \mathrm{m}^{3}$.

$C=\left[\begin{array}{ccccccc}25.2269 & & & & & \\ 10.5544 & 25.5865 & & & & \\ 10.6918 & 10.2232 & 25.5492 & & & \\ -0.1725 & -0.4684 & 0.7059 & 5.0638 & & \\ -0.6108 & -0.1236 & 0.7766 & -0.2024 & 5.7258 & \\ 0.0737 & -0.6231 & 0.4449 & 0.1602 & 0.1930 & 5.2015\end{array}\right]$

\subsection{Acquisition system}

During the process of wavefield modeling, the acquisition system is shown in Fig. 9. The circle point in the center represents the reflection point, and shots and receivers are located on the intersections between circles and radial lines. Concentric circles with equal distances represent different offsets, while the circle point represents shot and receiver.

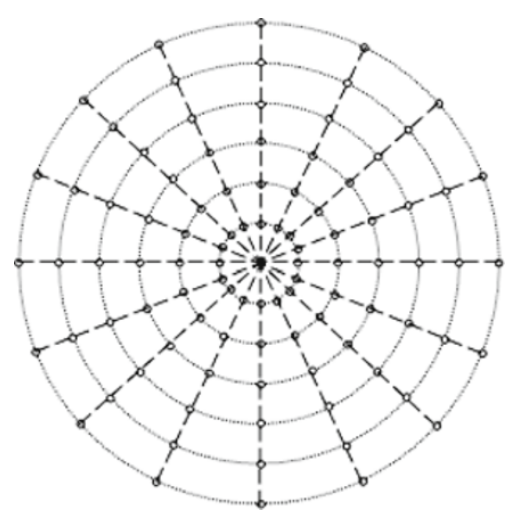

Fig. 9 Acquisition system

\subsection{Wavefield characteristics}

Using the parameters given in 4.1, staggered-grid highorder finite difference forward modeling is conducted. The characteristics of the azimuthal gathers in different offsets are analyzed, the wavefield features are provided in Fig. 10 and Fig. 11.

From Fig. 10 and Fig. 11, we can get the following conclusions. The wavefield features are rather complex for this quasi TTI anisotropic medium. For data with the same offset, the traveling time of reflection wave changes versus azimuth. The time difference between the minimum and the maximum traveling time of the P-P wave increases as the offset rises. Shear wave splitting exists when the shear wave propagates through the quasi TTI medium (as shown by the reflected shear wave $\mathrm{PS}_{1}, \mathrm{PS}_{2}$ and the direct shear wave $\mathrm{S}_{1}$, $\mathrm{S}_{2}$ ). From what we have mentioned above, we find that when the data processing is conducted, as the velocity in different azimuths varies, data processing toward different azimuths is necessary. The wavefield obtained through the forward modeling provides the input data for the following AVO inversion method, which acts as an important bridge in the fracture prediction flow shown in Fig. 1.

\section{Seismic modeling of a theoretical fracture model}

In order to further analyze the seismic responses of the fractured reservoir, a theoretical fracture model is designed, which contains two layers. The first layer is an isotropic medium, and the second layer is a monoclinic anisotropic medium. The model parameters and fracture parameters are shown in Table 2 and Table 3. Based on this model, seismic modeling is performed using the staggered-grid high-order finite difference method (Cerjan et al, 1985; Wang et al, 2005; 


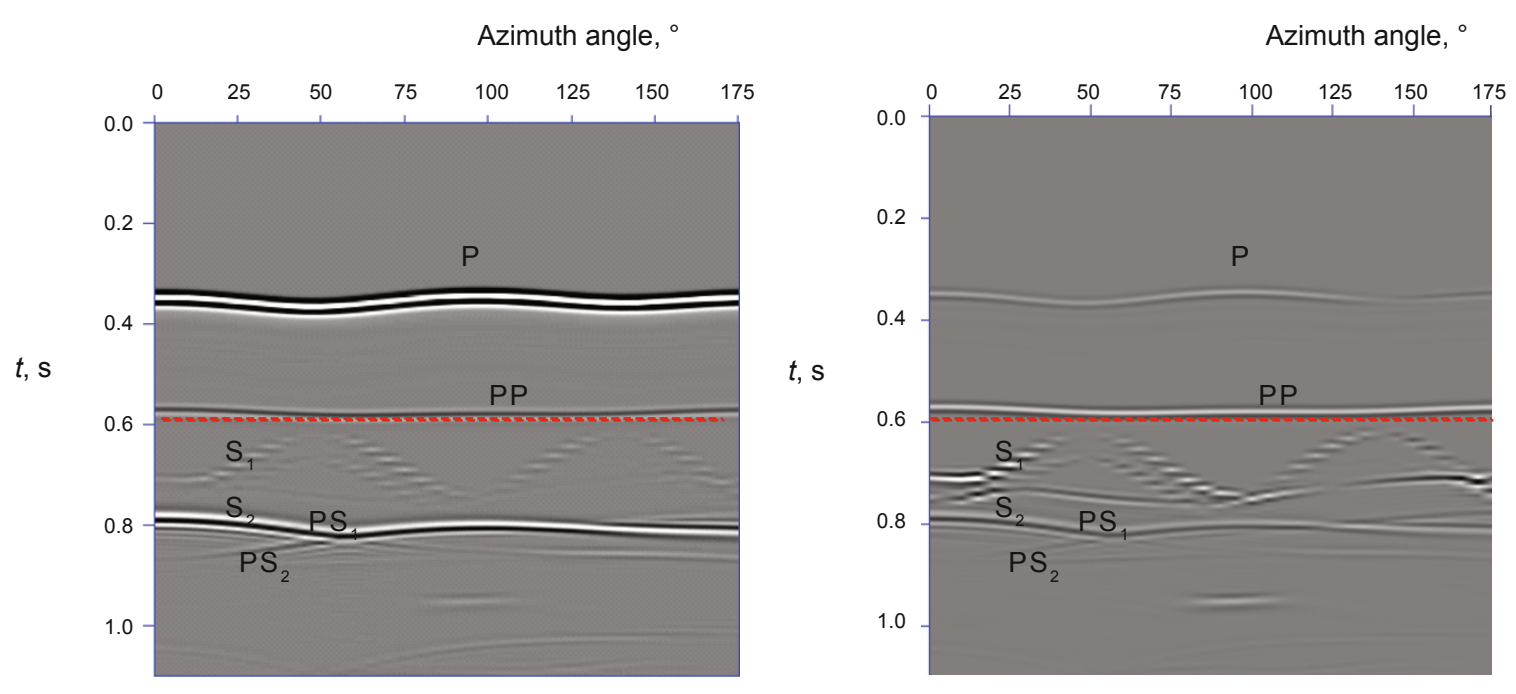

Fig. 10 Azimuthal gathers of $x$ and $z$ components at the offset of $1000 \mathrm{~m}$
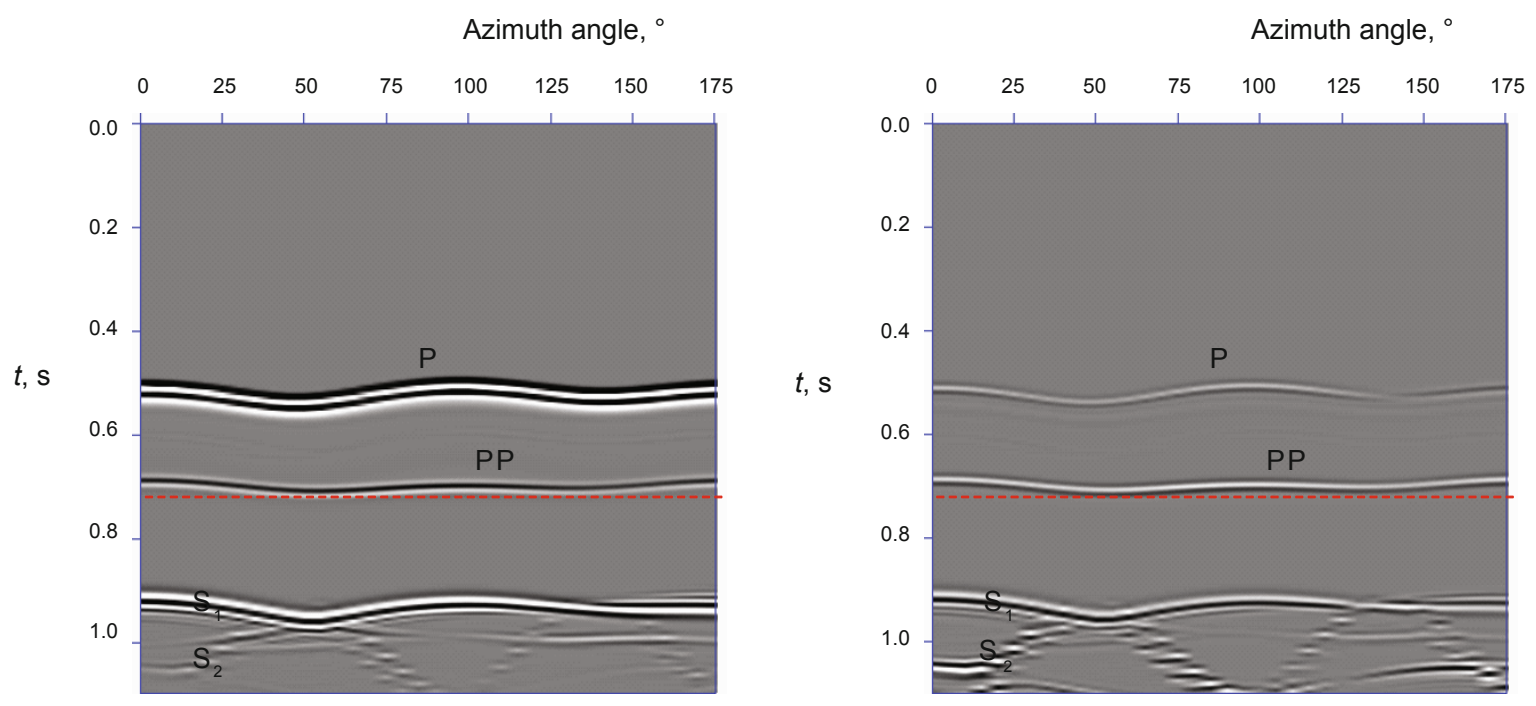

Fig. 11 Azimuthal gathers of $x$ and $z$ components at the offset of $1800 \mathrm{~m}$

Dong et al, 2000). The acquisition system is the same as shown in Fig. 9.

Table 2 Model 1 parameters

\begin{tabular}{|c|c|c|c|c|}
\hline Model & Layer & $V_{\mathrm{p}}, \mathrm{m} / \mathrm{s}$ & $V_{\mathrm{s}}, \mathrm{m} / \mathrm{s}$ & $\rho, \mathrm{kg} / \mathrm{m}^{3}$ \\
\hline \multirow{2}{*}{$\begin{array}{c}\text { Model } 1 \\
\text { (clastic rock) }\end{array}$} & isotropy & 2800.0 & 1800.0 & 2300.0 \\
\hline & $\begin{array}{c}\text { Layer } 2 \\
\text { (base-rock) }\end{array}$ anisotropy & 3500.0 & 2000.0 & 2300.0 \\
\hline
\end{tabular}

Table 3 Fracture parameters

\begin{tabular}{ccccc}
\hline & Normal direction, $^{\circ}$ & Radius, $\mathrm{m}$ & Aspect ratio & Density \\
\hline 1 & 0 & 1.0 & 0.001 & 0.01 \\
2 & 60 & 1.0 & 0.001 & 0.1 \\
\hline
\end{tabular}

\subsection{Wavefield of a monoclinic medium}

We select the parameters in model 1 (Table 2). Fig. 12 shows the angle gathers with different offsets of the model. From $0^{\circ}$ to $180^{\circ}$, the reflection travel-time curves show negative sine characteristics. The longest travel-time of the PP wave appears at about $55^{\circ}$ and the shortest appears at about $145^{\circ}$. With the offset increasing, the difference between the shortest and the longest increases. At about $55^{\circ}$, the PS wave travels at the slow $\mathrm{S}$ wave velocity and there is only $\mathrm{PS}_{2}$ whose energy is very strong. At about $145^{\circ}$, the PS wave travels at the fast $\mathrm{S}$ wave velocity and there is only $\mathrm{PS}_{1}$ whose energy is also very strong. And at other azimuth angles, $\mathrm{PS}_{1}$ and $\mathrm{PS}_{2}$ exist at the same time, but their energies are weak. So converted waves are sensitive to fractures and it is feasible to use the converted waves to detect the fractures. Moreover, the azimuth must be considered during processing the azimuth- 
angle gathers.

\subsection{Influence of fluids}

We still select the parameters in model 1 (Table 2). For analyzing the fluid influence, we respectively use oil and water to fill the fractures. Fig. 12(b) and Fig. 13 show that: from $0^{\circ}$ to $180^{\circ}$, when fractures are filled with oil or water, the PP reflection travel-time curves also have negative sine characteristics. Regardless of whether the fractures are filled with oil or water, the PP, $\mathrm{PS}_{1}$ and $\mathrm{PS}_{2}$ show similar characteristics. When fractures are filled with oil or water, the difference between the longest and shortest travel time is smaller than that when fractures are filled with gas. In summary, when the fractures are filled with gas, the model displays obvious azimuth-anisotropy, while when filled with oil or water, the azimuth-anisotropy is weak.

\subsection{Influence of base-rock}

For studying the base-rock influence, the model is changed from model 1 to model 2 (Table 4). The base-rock of the fractured reservoir is changed from clastic rock to carbonate rock, and the fracture parameters are kept the same (Table 3). Fig. 14 shows the modeling results of model 2 with different fluids in fractures. Comparing with Fig. 12(b), Fig. 13 and Fig. 14, we find that when the fractures are filled with the same fluid, but the base rock is different, the difference between the shortest and the longest reflection travel-time decreases from clastic base-rock to carbonate base-rock. Therefore it is easier to distinguish gas from oil and water in clastic rock than in carbonate rock.

Table 4 Model 2 parameters

\begin{tabular}{cccccc}
\hline Model & \multicolumn{2}{c}{ Layer } & $V_{\mathrm{p}}, \mathrm{m} / \mathrm{s}$ & $V_{\mathrm{s}}, \mathrm{m} / \mathrm{s}$ & $\rho, \mathrm{kg} / \mathrm{m}^{3}$ \\
\hline $\begin{array}{c}\text { Model 2 } \\
\text { (carbonate rock) }\end{array}$ & $\begin{array}{c}\text { Layer 1 } \\
\text { Layer 2 } \\
\text { (base-rock) }\end{array}$ & isotropy & 4800.0 & 3200.0 & 2600.0 \\
\hline
\end{tabular}

\section{The reflection coefficients characteristics of a fractured reservoir}

Spherical divergence exists when performing seismic modeling, so it is not suitable for amplitude analysis. To do this more easily, the Zoeppritz equation is applied in this paper to analyze the reflection coefficient characteristics of a
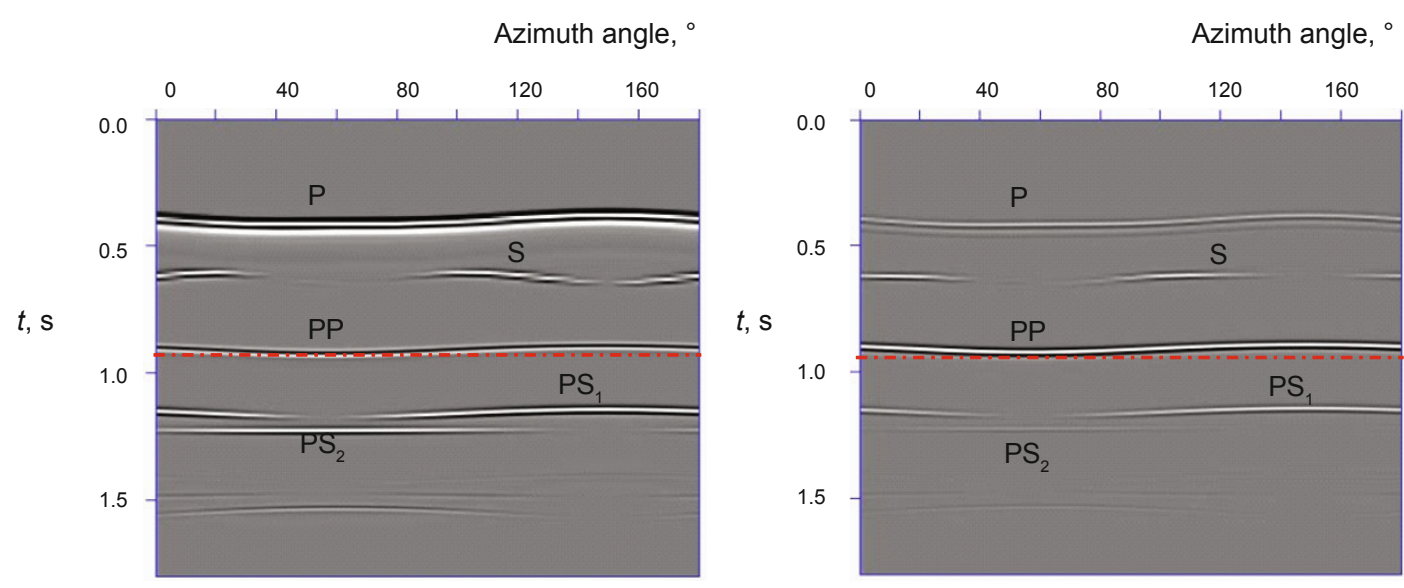

(a) Angle gathers of $x$ and $z$ components at offset $1200 \mathrm{~m}$
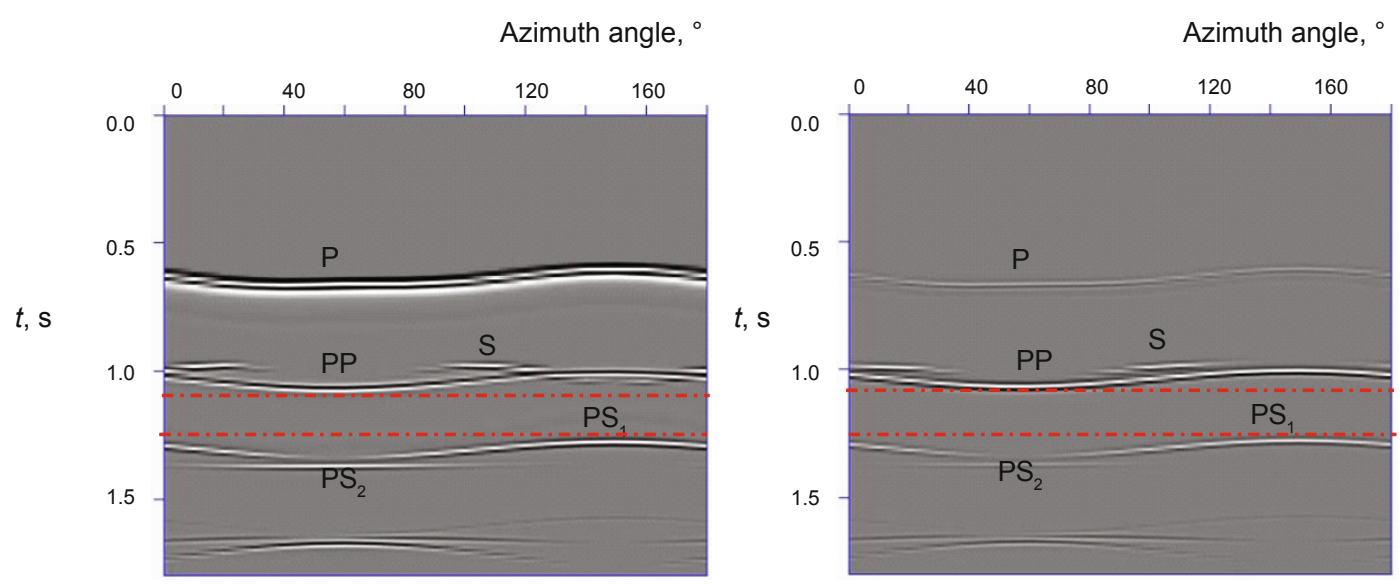

(b) Angle gathers of $x$ and $z$ components at offset $1980 \mathrm{~m}$

Fig. 12 Angle gathers of $x, z$ components at different offsets in clastic rock 


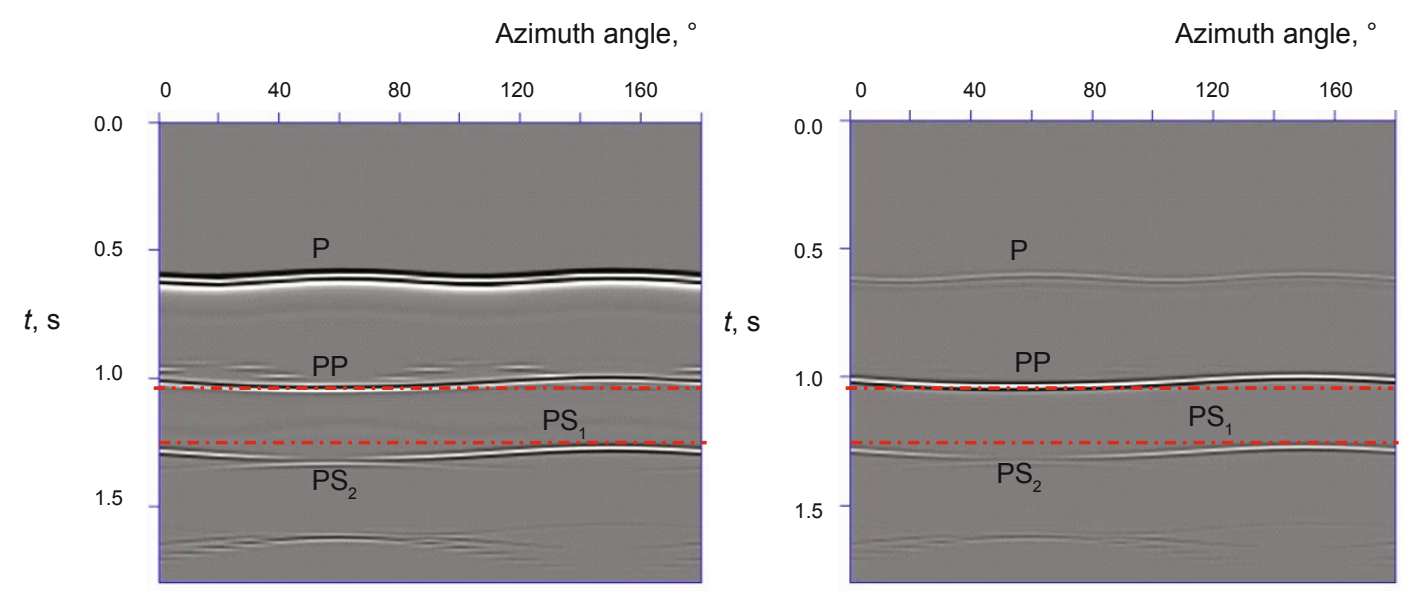

(a) Azimuth-angle gathers $x$ and $z$ components with fractures filled with oil

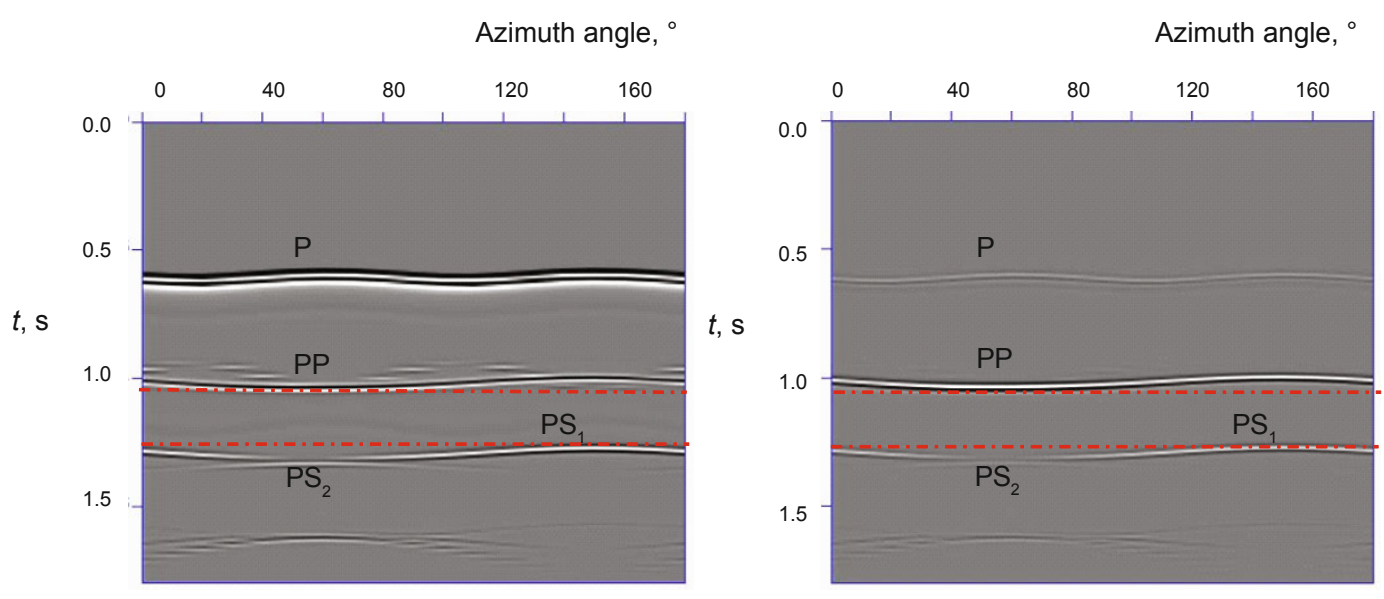

(b) Azimuth-angle gathers $x$ and $z$ components with fractures filled with water

Fig. 13 Azimuth-angle gathers with 1980m offset in clastic rock

fractured reservoir. Reflection and transmission are produced when a seismic wave arrives at the elastic interface. The seismic wave is considered as a plane wave. According to Huygen's principle, the incidence, reflection, and transmission waves should satisfy Snell's Law as well as the displacement continuity and the stress continuity on the boundary ( $\mathrm{Wu}$, 2006). Then, the Zoeppritz equation which describes the reflection coefficient and transmission coefficient under anisotropic condition can be obtained. For example, the Zoeppritz equation of a compression wave under anisotropic conditions is shown as follows:

$$
\left[\begin{array}{cccc}
\sin \theta_{\mathrm{PR}} & \cos \theta_{\mathrm{SR}} & -\sin \theta_{\mathrm{PT}} & \cos \theta_{\mathrm{ST}} \\
\cos \theta_{\mathrm{PR}} & -\sin \theta_{\mathrm{SR}} & \cos \theta_{\mathrm{PT}} & \sin \theta_{\mathrm{ST}} \\
A_{1} & A_{2} & A_{3} & A_{4} \\
A_{5} & A_{6} & A_{7} & A_{8}
\end{array}\right]\left[\begin{array}{c}
R_{\mathrm{PP}} \\
R_{\mathrm{PS}} \\
T_{\mathrm{PP}} \\
T_{\mathrm{PS}}
\end{array}\right]=\left[\begin{array}{c}
-\sin \theta_{\mathrm{PR}} \\
\cos \theta_{\mathrm{PR}} \\
B_{1} \\
B_{2}
\end{array}\right]
$$

where, $A_{i}$ and $B_{i}$ are function of elastic parameters, incidence angle and transmission angle on both sides of the interface. $R_{\mathrm{PP}}$ and $R_{\mathrm{PS}}$ respectively represent the reflection coefficients of the $\mathrm{P}$-wave and converted S-wave. $T_{\mathrm{PP}}$ and $T_{\mathrm{PS}}$ respectively represent the transmission coefficients of the P-wave and converted S-wave.

\subsection{Characteristics of reflection coefficients with incident angles}

We select the model 1 (Table 2) and calculate the reflection coefficients (Fig. 15). From Fig. 15, the following conclusions can be derived. For a certain azimuth, the reflection coefficient changes with the incident angle (denoted by the slowness), and if the azimuth angle changes, the reflection coefficient also changes. The lines on the $30^{\circ}$ and $120^{\circ}$ are respectively perpendicular and parallel to the bisecting line of the included angle between the two groups of fractures, and the P-wave velocity is smallest on $30^{\circ}$ and is biggest on $120^{\circ}$, so the reflection coefficient is different obviously from the slowness of $0.25 \mathrm{~s} / \mathrm{m}$ to the slowness of $0.35 \mathrm{~s} / \mathrm{m}$. The lines on the $90^{\circ}$ and the $150^{\circ}$ are respectively parallel to the fracture strikes, and the corresponding reflection coefficients are different because the fracture intensities are not the same for fractures in these two directions. Fig. 15(e) displays the differences of the PP reflection coefficients on different azimuth angles and the differences on middle offsets are more 


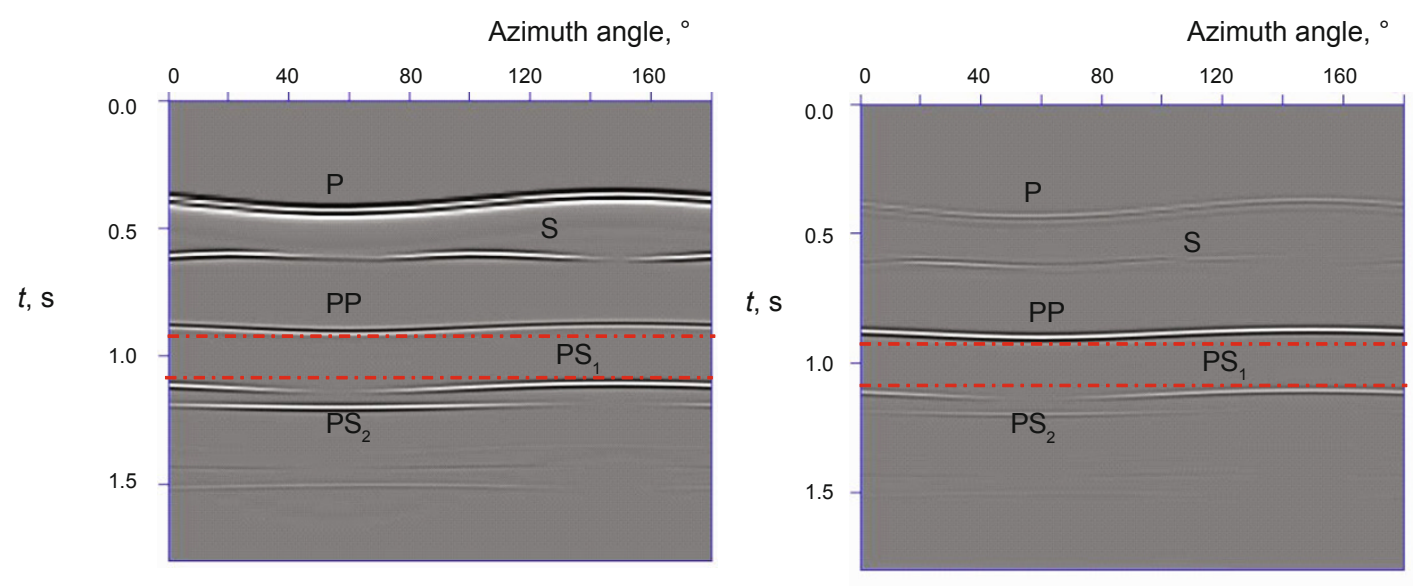

(a) Azimuth-angle gathers $x$ and $z$ components with fractures filled with gas

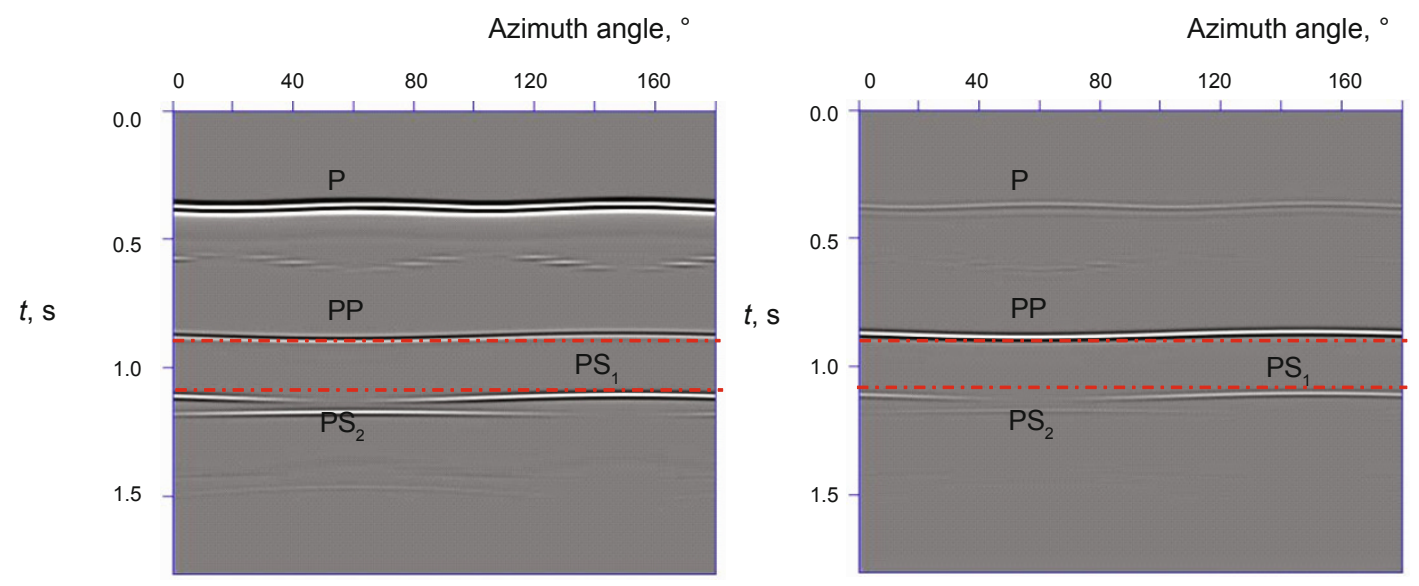

(b) Azimuth-angle gathers $x$ and $z$ components with fractures filled with oil

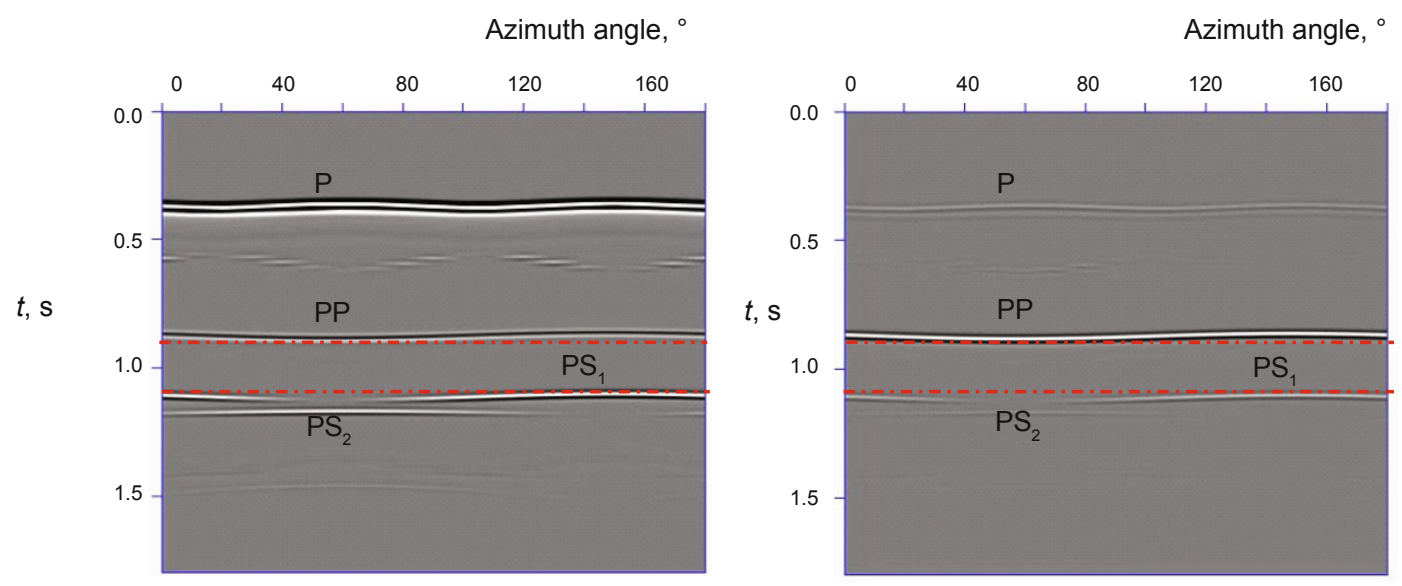

(c) Azimuth-angle gathers $x$ and $z$ components with fractures filled with water

Fig. 14 Azimuth-angle gathers with 1980m offset in carbonate rock

obvious than those on near offsets.

\subsection{Characteristics of reflection coefficients with azimuth angles}

Fig. 16 shows the reflection coefficients changing with the azimuth angles for the model 1 (Table 2). The reflection coefficient changes with the azimuth angle for a certain fixed incident angle. Slowness of $0.198 \mathrm{~s} / \mathrm{m}, 0.286 \mathrm{~s} / \mathrm{m}, 0.358 \mathrm{~s} / \mathrm{m}$ and $0.418 \mathrm{~s} / \mathrm{m}$ are selected as examples, the PP reflection coefficient changes gently at $0.198 \mathrm{~s} / \mathrm{m}, 0.358 \mathrm{~s} / \mathrm{m}$ and 0.418 $\mathrm{s} / \mathrm{m}$, while the PP reflection coefficient changes dramatically at $0.286 \mathrm{~s} / \mathrm{m}$. The azimuth AVO characteristics exist on the top interface of the fractured reservoirs, and we might be able to use this characteristic to invert fracture development. 


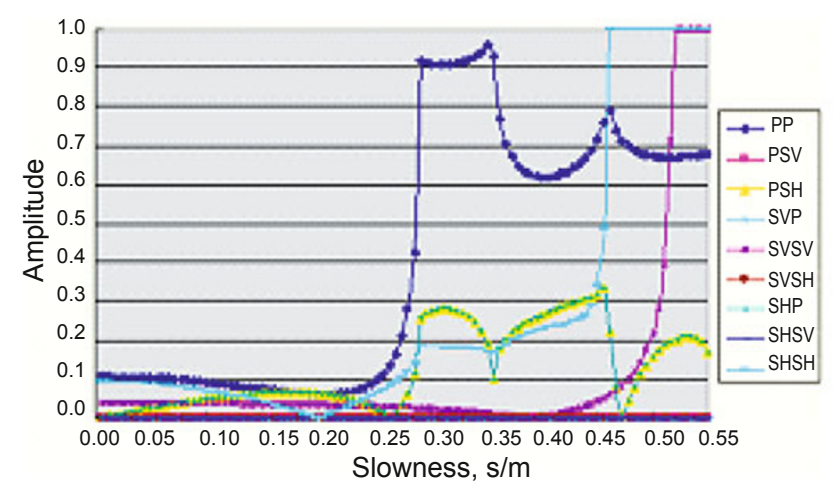

(a) Azimuth angle of $0^{\circ}$

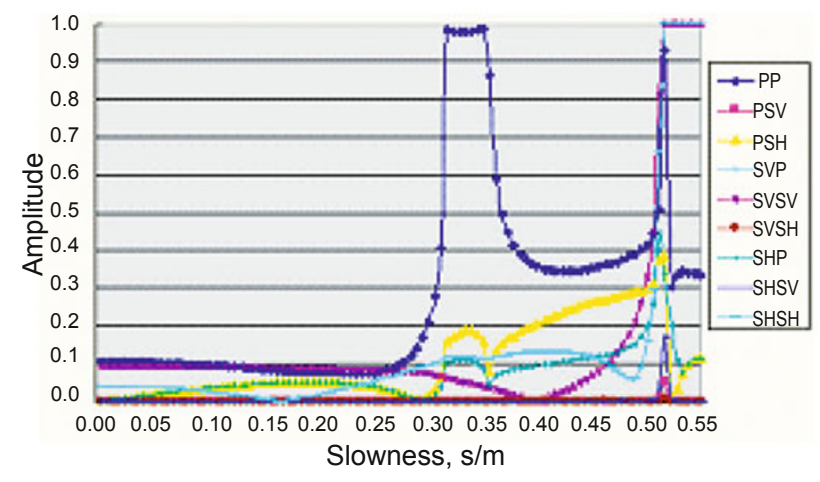

(c) Azimuth angle of $90^{\circ}$

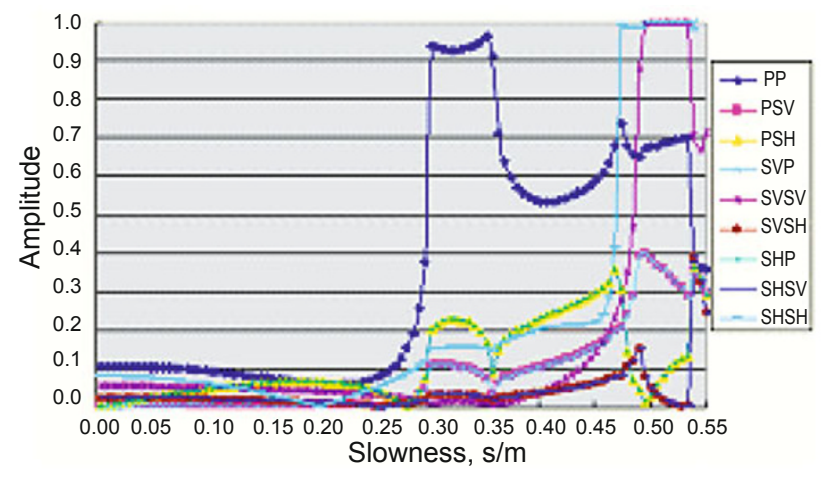

(b) Azimuth angle of $30^{\circ}$

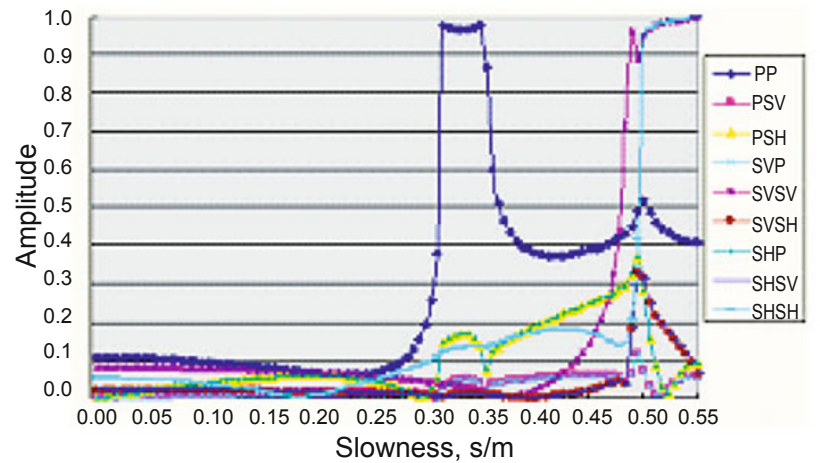

(d) Azimuth angle of $120^{\circ}$

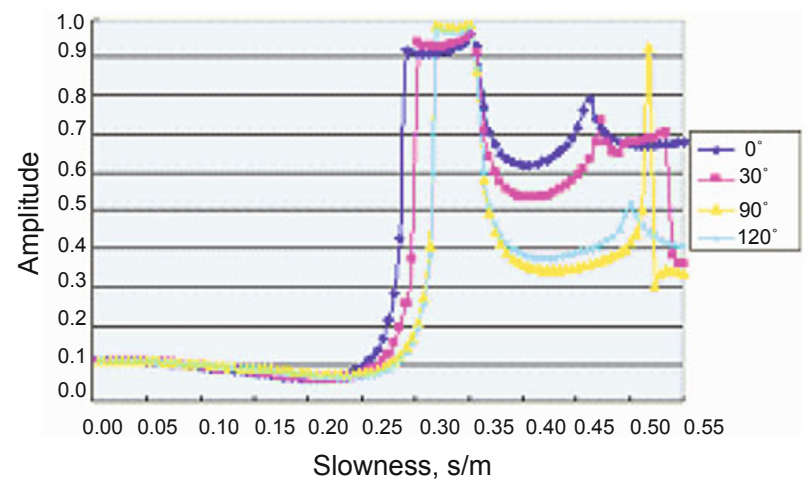

(e) PP reflection coefficients

Fig. 15 Diagrams showing the reflection coefficients changing with incident angles in clastic reservoir

\subsection{Influence of fluid properties}

For analyzing the influence of fluid properties on reflection coefficients, the fractures of the model 1 (Table 2) are filled respectively with oil and water. At the $30^{\circ}$ azimuth, selecting the slowness area from $0.05 \mathrm{~s} / \mathrm{m}$ to $0.25 \mathrm{~s} / \mathrm{m}$, when the fractures are filled with gas (Fig. 15(b)), the PP reflection coefficient decreases apparently almost to 0.06 . However, when the fractures are filled with oil or water (Fig. 17(a) and Fig. 17(b)), the PP reflection coefficient decreases apparently almost to 0.09 . At the slowness from $0.20 \mathrm{~s} / \mathrm{m}$ to $0.30 \mathrm{~s} / \mathrm{m}$, the PP reflection coefficient when filled with gas is smaller than that when filled with oil or water. The law is the same at the $120^{\circ}$ azimuth. For example, the PP reflection coefficient is 0.2 at $0.3 \mathrm{~s} / \mathrm{m}$ when filled with gas, while the PP reflection coefficient is 0.2 at $0.28 \mathrm{~s} / \mathrm{m}$ when filled with oil or water. From Fig. 17(a) and Fig. 17(b), we find that when the azimuth is $30^{\circ}$ or $120^{\circ}$, the PP reflection coefficient when filled with oil is similar with that when filled with water. Therefore, it is very difficult to distinguish oil from water from the reflection coefficient.

\subsection{Influence of base-rocks}

For analyzing the influence of the base-rocks, we select the model 2 (Table 4) with carbonate rock as base-rock. As shown in Fig. 18, the curves are different when filled with gas compared to filled with oil or water at $30^{\circ}$ and $120^{\circ}$. So we can distinguish gas from oil and water, but oil and water are almost indistinguishable from the PP reflection coefficient. 


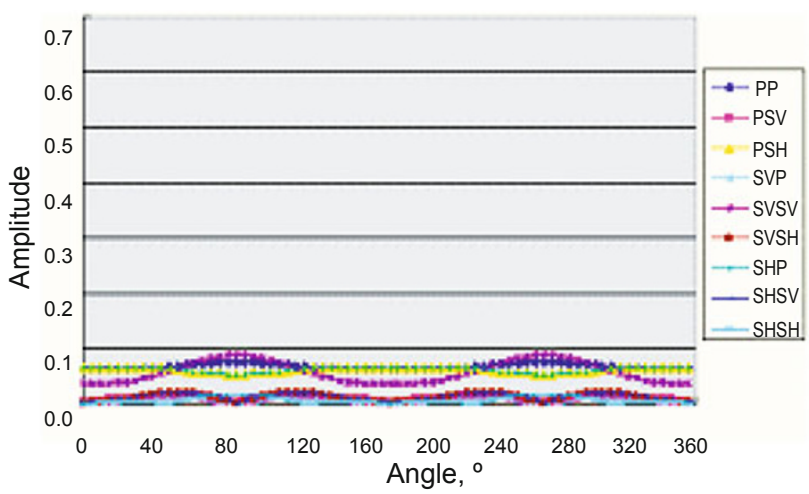

(a) Slowness $0.198 \mathrm{~s} / \mathrm{m}$

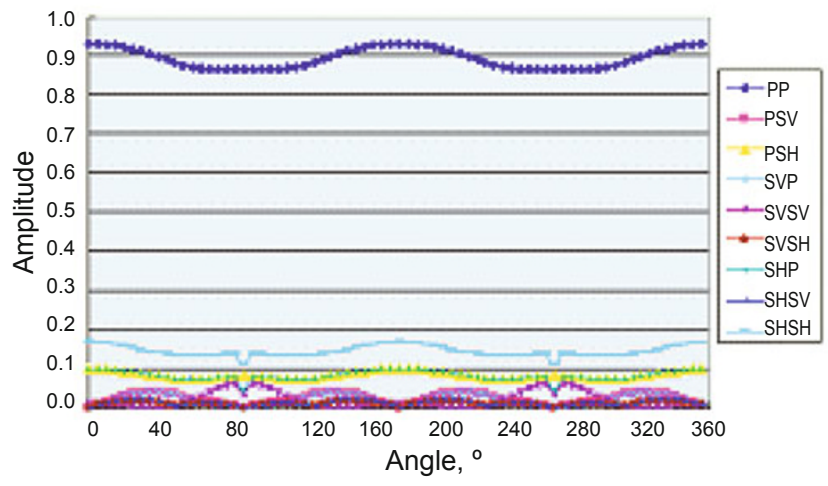

(c) Slowness $0.358 \mathrm{~s} / \mathrm{m}$

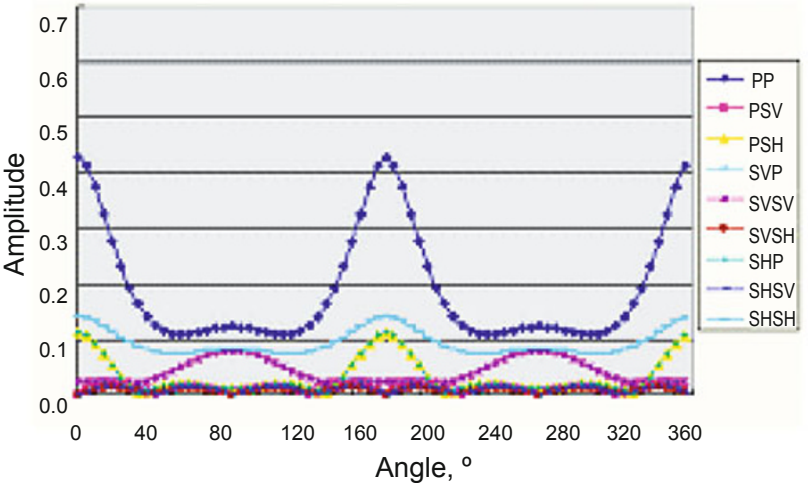

(b) Slowness $0.286 \mathrm{~s} / \mathrm{m}$

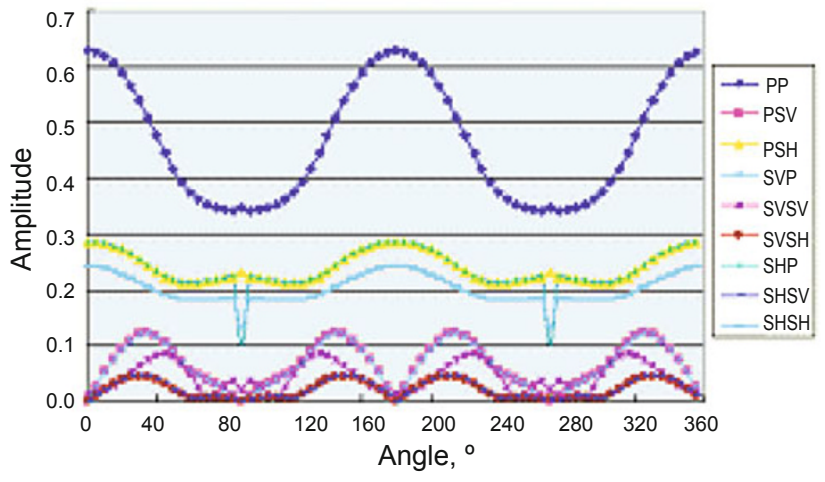

(d) Slowness $0.418 \mathrm{~s} / \mathrm{m}$

Fig. 16 Diagrams showing the reflection coefficients changing with azimuth angles in clastic reservoir
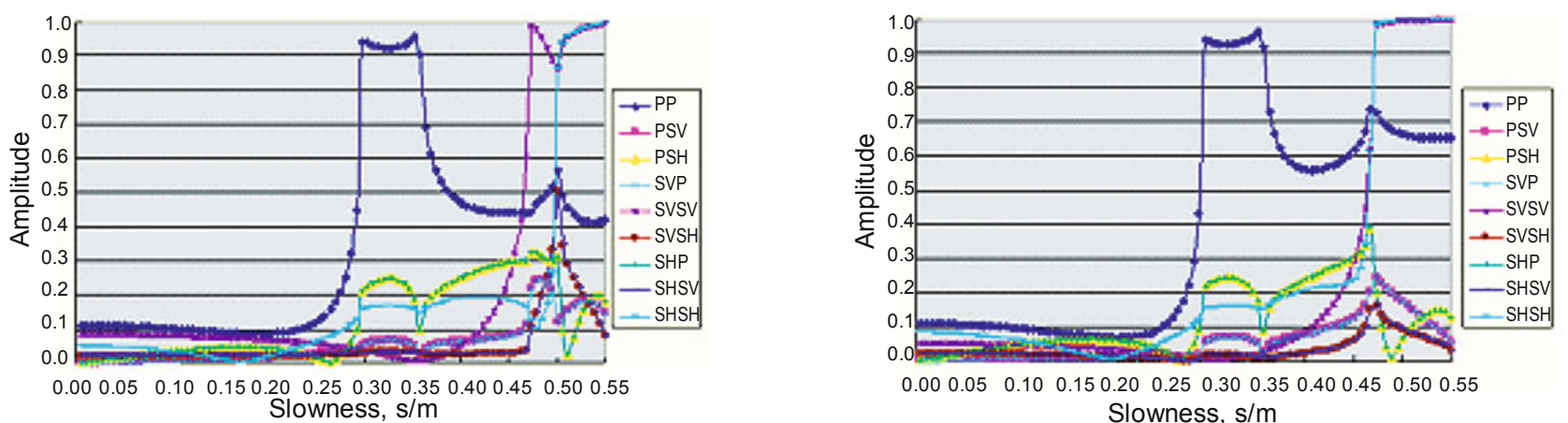

(a) Reflection coefficients at $30^{\circ}$ and $120^{\circ}$ azimuth with fractures filled with oil
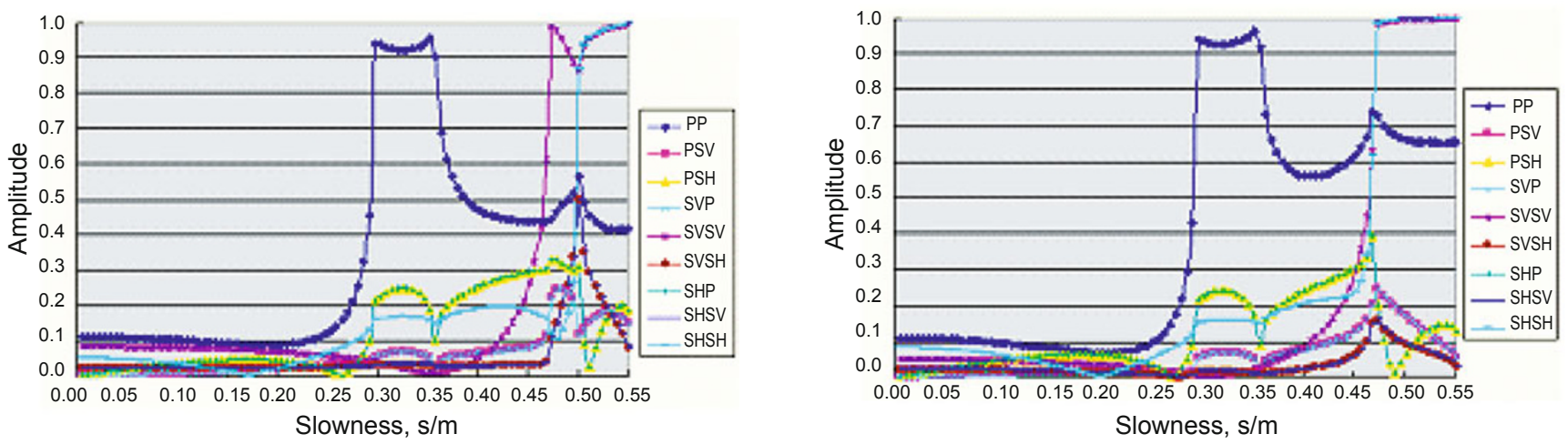

(b) Reflection coefficients at $30^{\circ}$ and $120^{\circ}$ azimuth with fractures filled with water

Fig. 17 Diagrams showing the reflection coefficients changing with slowness with fractures filled with different fluids in clastic reservoir 

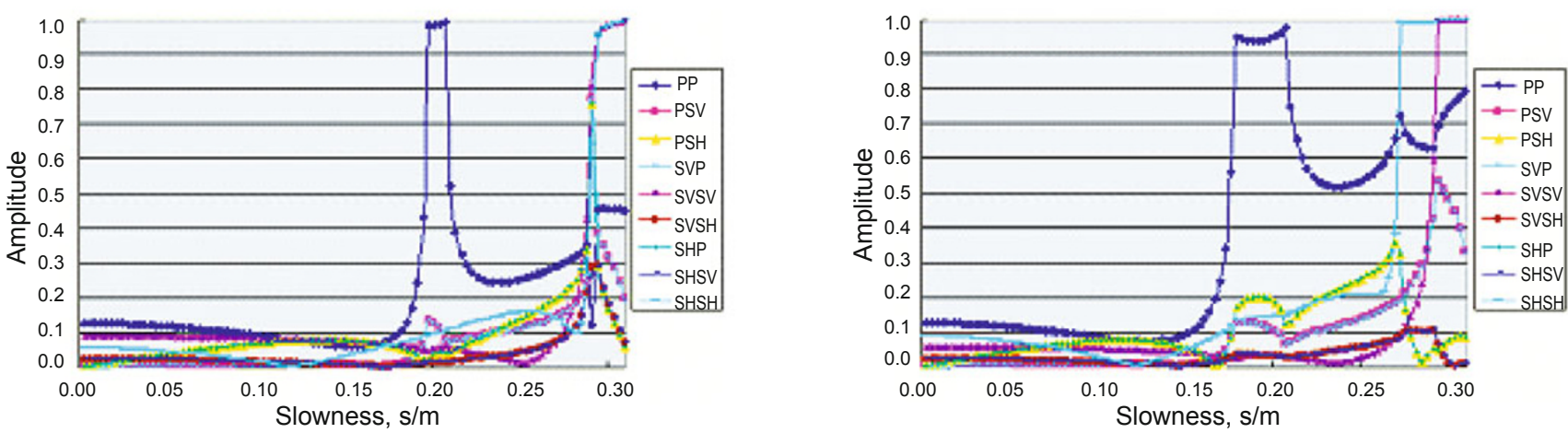

(a) Reflection coefficients at $30^{\circ}$ and $120^{\circ}$ azimuth with fractures filled with gas
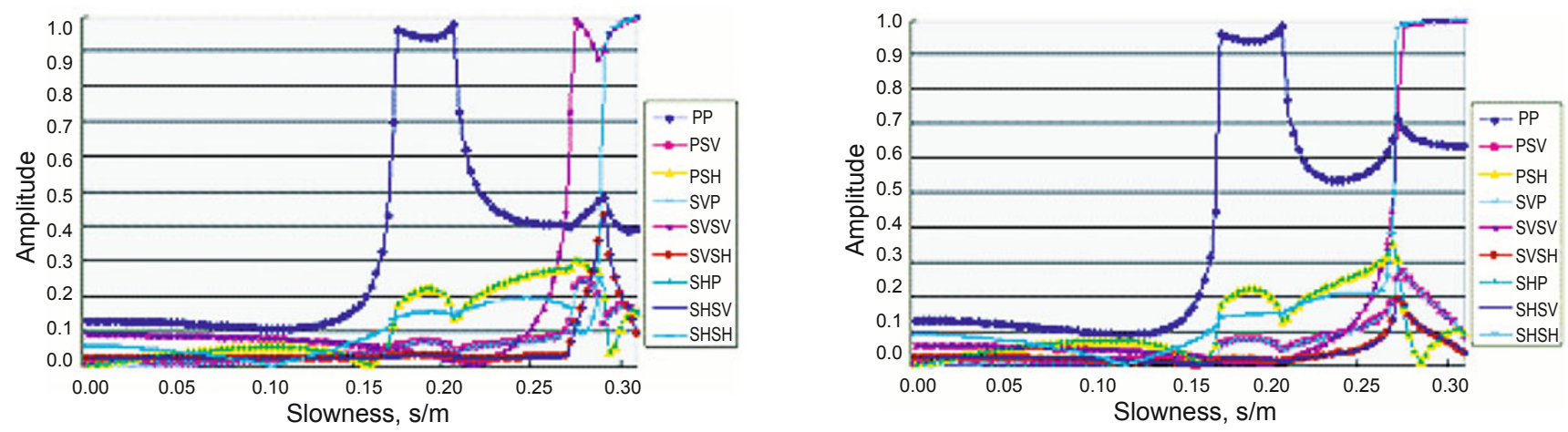

(b) Reflection coefficients at $30^{\circ}$ and $120^{\circ}$ azimuth with fractures filled with oil
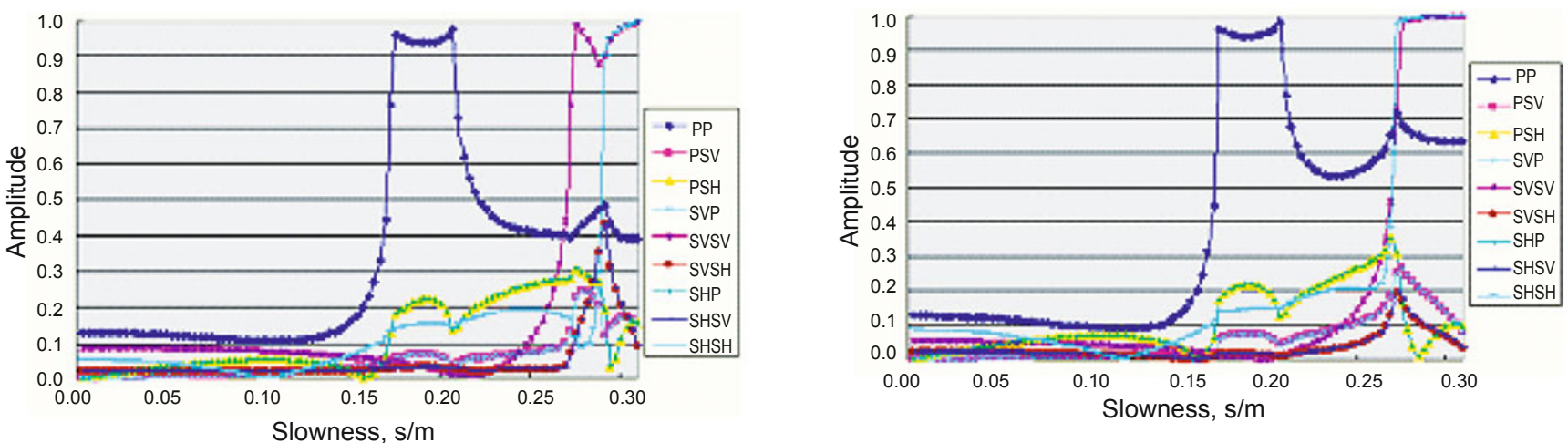

(c) Reflection coefficients at $30^{\circ}$ and $120^{\circ}$ azimuth with fractures filled with water

Fig. 18 Diagrams showing reflection coefficients changing with slowness with fractures filled with different fluids in carbonate reservoir

\section{Conclusions}

1) The geological, seismic and well log data respectively describe the fractures at different scales, and an integration of the three can be used to build up the fracture models by the DFN method and the fracture parameters can be calculated. The equivalent medium theory uses the fracture parameters to compute the elastic coefficients of the anisotropic medium, which is an important bridge to connect the microscopic fractures and macroscopic reservoir modeling.

2) Using the real geological, seismic and well longing data, the DFN model is built to calculate the equivalent elastic coefficient. The wavefield forward modeling is conducted using the staggered-grid high-order finite difference method.
The modeling result shows that for data with the same offset, the traveling time of the reflection wave changes with the azimuth. Additionally, the time difference between the minimum and the maximum traveling time of the P-P wave increases as the offset rises. Shear wave splitting exists when a shear wave propagates through the quasi TTI medium. The wavefield through the forward modeling not only helps us to understand the seismic response of the fractured reservoirs, but also provides the input data for the study of the fracture inversion method.

3) From an analysis of the monoclinic anisotropy, we learn some characteristics of the reflection coefficients, the slowness and the azimuth angles. The PP reflection coefficients in middle offsets are sensitive to the fractures, so 
the middle offsets are better than the others when inverting the fractures. The seismic data are more sensitive to gas than oil or water, so gas can be distinguished from oil and water, while oil can not be distinguished from water. When the baserocks are different, it is much easier to distinguish the fluids in the clastic rock than in carbonate rock. Azimuth anisotropy can be used in detecting fractured reservoirs. Theoretically, the acquisition system for anisotropy detection should be fullazimuth and have equal fold. Data processing toward different azimuth is necessary as the velocities in different azimuth are not the same.

\section{Acknowledgements}

The work is co-supported by the National Basic Research Program of China (Grant No.2011CB201103) and the National Science and Technology Major Project (Grant No.2011ZX05004003). The authors would like to thank the Laboratory for Integration of Geology and Geophysics (LIGG) at China University of Petroleum for the permission to publish this work and the Tarim Oilfield Co., PetroChina for their help in providing field data. Also, Shell Co. is thanked for the financial assistant to the first author.

\section{References}

Cerjan C, Kosloff D, Kosloff R, et al. A nonreflecting boundary condition for discrete acoustic and elastic wave equations. Geophysics. 1985. 50(4): 705-708

Crampin S. Effective anisotropic elastic constants for wave propagation through cracked solids. Geophysical Journal of the Royal Astronomical Society. 1984. 76(1): 135-145

Dong L G, Ma Z T, Cao J Z, et al. A staggered-grid high-order difference method of one-order elastic wave equation. Chinese Journal of Geophysics. 2000. 43(3): 411-419 (in Chinese)

Hudson J A. A higher order approximation to the wave propagation constants for a cracked solid. Geophysical Journal of the Royal Astronomical Society. 1986. 87(1): 265-274
Luo J H, Zhou X Y, Qiu B, et al. Mesozoic-Cenozoic five tectonic events and their petroleum geologic significances in the West Tarim Basin. Petroleum Exploration and Development. 2005. 32(1): 18-22 (in Chinese)

Tsvankin I. Seismic Signatures and Analysis of Reflection Data in Anisotropic Media. Amsterdam: Elsevier Science. 2005. 14-252

Wang D L, He Q D and Han L G. Multi-azimuth three-component surface seismic modeling for cracked monoclinic media. Chinese Journal of Geophysics. 2005. 48(2): 386-393 (in Chinese)

Wang S M, Jin Z J and Xie Q L. Transforming effect of deep fluids on carbonate reservoirs in the well TZ45 region. Geological Review. 2004. 50(5): 543-547 (in Chinese)

Wang Z Y, Li Y P, Chen J S, et al. Characters of atmospheric diagenetic lens along middle-late Ordovician carbonate shelf margin in central Tarim area. Chinese Journal of Geology. 2002. 37(S1): 152-160 (in Chinese)

Wang Z Y, Yan W, Zhang Y F, et al. Diagenesis and porosity evolution of upper Ordovician platform margin reefs and grain banks reservoirs in the Tazhong area. Xinjiang Geology. 2007. 25(3): 288-290 (in Chinese)

Wei G Q, Jia C Z, Song H Z, et al. Ordovician structural-depositional model and prediction for profitable crack reservoirs of carbonate rock in the Tazhong area, Tarim Basin. Acta Sedimentologica Sinica. 2000. 18(3): 408-413 (in Chinese)

Wu G C. Seismic Wave Propagation and Imaging in an Anisotropic Medium. Dongying: China University of Petroleum Press. 2006. 43156 (in Chinese)

Yang H J, Liu S, Li Y P, et al. Analysis of the middle-upper Ordovician carbonate reservoirs in the Tazhong area. Marine Origin Petroleum Geology. 2000. 5(1-2): 73-83 (in Chinese)

Zhao Z J, Wang Z M, Wu X N, et al. Genetic types and distribution forecast of available carbonate reservoirs in Ordovician in the central area of Tarim Basin. Petroleum Geology and Experiment. 2007. 29(1): 40-46 (in Chinese)

Zhu D Y, Hu W X, Song Y C, et al. Fluoritization in Tazhong 45 reservoir: characteristics and its effect on the reservoir bed. Acta Petrologica et Mineralogica. 2005. 24(3): 205-215 (in Chinese)

(Edited by Hao Jie) 